\title{
Klebsiella pneumoniae Invasive Syndrome
}

\author{
Vasco Evangelista, Cristiana V. Gonçalves, Raquel Almeida, Célia Henriques, António Martins Baptista, \\ José Pimenta da Graça, José Lomelino Araújo \\ Department of Medicine, Hospital Beatriz Ângelo, Loures, Portugal
}

How to cite this article: Evangelista V, Gonçalves CV, Almeida R, Heriques C, Baptista AM, Pimenta da Graca J, Lomelino Araújo J. Klebsiella pneumoniae invasive syndrome. EJCRIM 2018;5: doi:10.12890/2018_000800.

Conflicts of Interests: The Authors declare that there are no competing interests.

This article is licensed under a Commons Attribution Non-Commercial 4.0 License

\section{ABSTRACT}

Klebsiella pneumoniae invasive syndrome (KPIS) is a rare clinical condition characterized by primary liver abscess associated with metastatic infection. Most case reports are from Southeast Asia, with only one case described in Portugal. The Authors present the case of a 44-year-old man with a history of fever, dry cough and cervicalgia. A thoracic computed tomography (CT) scan showed multiple pulmonary and hepatic nodules, suggestive of metastatic malignancy. Both blood cultures and bronchoalveolar lavage were positive for Klebsiella pneumoniae. Imaging studies were repeated during his hospital stay, showing a reduction in both number and volume of identified lesions, thus revealing their infectious nature. This case illustrates how much this entity can mimic other illnesses.

\section{LEARNING POINTS}

- Klebsiella pneumoniae invasive syndrome is emerging as a global disease.

- The imaging-led diagnosis of neoplasia was proved incorrect and could have been deleterious for the patient.

- The lack of diagnostic suspicion can lead to shorter antibiotic treatment regimens, therefore compromising the patient's full recovery.

\section{KEYWORDS}

Klebsiella pneumoniae, invasive syndrome, liver abscess

\section{INTRODUCTION}

Klebsiella pneumoniae is a Gram-negative bacillus with worldwide distribution that usually causes respiratory and urinary tract infections, both in nosocomial and community settings. The first cases of primary liver abscess with metastatic dissemination in patients infected by K. pneumoniae were described in the 1980s in anecdotal reports from Southeast Asia, namely Taiwan ${ }^{[1,2]}$. Since then, K. pneumoniae has emerged as the primary agent for this community-acquired infection, with hundreds of cases being reported in the last three decades in all Asian countries ${ }^{[3]}$.

KPIS represents a rare clinical condition in the West characterized by primary liver abscess associated with bacteremia and metastatic infections, including brain abscess, suppurative meningitis, endophthalmitis and necrotizing fasciitis. The source of invasive K. pneumoniae infection in individual patients remains unknown. The disease being community acquired, patients are usually immunocompetent and have no underlying intestinal or hepatobiliary disease. The invasive nature of these strains is due to specific virulence characteristics, such as the hypermucoviscosity phenotype related to $\mathrm{K} 1$ and $\mathrm{K} 2$ capsular serotypes and certain genetic plasmids (mucoviscosity-associated gene $\mathrm{A}$ (magA), and regulator of mucoid phenotype $A(\operatorname{rmpA}))^{[4]}$. 
These specific hypermucoviscous strains have been found to be susceptible to most antibiotics and therefore relatively easy to treat, although requiring prolonged treatment regimens. Mortality rates range from $4 \%$ to $11 \%$, reflecting the diagnostic delay due to lack of suspicion in Western physicians. In Europe, isolated reports have been described in countries such as Spain, Sweden, Ireland ${ }^{[5]}$ and France, with only one case reported in Portugal.

\section{CASE REPORT}

We present the case of a 44-year-old Portuguese man with pulmonary tuberculosis diagnosed two years earlier and treated accordingly for 6 months, and also a history of chronic alcohol-related pancreatitis with pancreatic insufficiency and insulin-dependent diabetes. His medication was Lantus ${ }^{\circledR}$ insulin. Family history was not relevant and there was no other drug use, travelling history or animal contact reported.

The patient presented to the emergency department with a 2-week history of fever $\left(39^{\circ} \mathrm{C}\right)$, dry cough, mucoid rhinorrhea and cervicalgia. Physical examination showed normal mental status and slight dehydration. He was eupneic while breathing room air, $\mathrm{SpO}_{2} 95 \%$, with no signs of breathing difficulty; normotensive, BP 106/93 mmHg, HR 97 bpm; and febrile (tympanic temperature of $38^{\circ} \mathrm{C}$ ). Cardiopulmonary auscultation revealed bilateral basal crackles. His abdomen was soft with palpable hepatomegaly. An intense cervicalgia was evident with painful palpation of $\mathrm{C} 4$ and $\mathrm{C} 5$ spinous processes causing immobilization. There were no neurologic findings on examination.

Laboratory tests showed normocytic normochromic anemia (Hb 13.0 g/dL, MCV 86 fL), raised inflammatory markers (20,600/ $\mathrm{LL}$ leukocytes, relative neutrophilia (89.9\%) and C-reactive protein $20.24 \mathrm{mg} / \mathrm{dL})$, hyperglycemia (301 mg/dL), normal renal and hepatic markers, raised $\mathrm{LDH}$ (288 UI/L) and hyponatremia (125 mmol/L).

Chest radiography revealed numerous bilateral nodular hypotransparencies, as well as cavitation on the right middle lobe. Cervical radiography was normal. Community-acquired pneumonia was diagnosed and empiric antimicrobial therapy with amoxicillin/clavulanic acid and azithromycin was started after blood cultures withdrawn. The patient was then admitted to an internal medicine ward.

Diagnostic work-up included several complementary tests summarized in Table 1. A thoracic CT scan showed multiple pulmonary nodular lesions suggestive of metastases. It also identified a juxtapleural nodular condensation on the right lower lobe with spiculated margins and eccentric cavitation, acknowledged as the probable primary tumor. Appreciation of the subdiaphragmatic segments also revealed multiple nodular hypodense liver lesions suggestive of metastatic malignancy with a confluent pattern (Fig. 1). Given the persistence of refractory cervicalgia, a cervical MRI was performed, which suggested secondary epidural infiltration (Fig. 2).

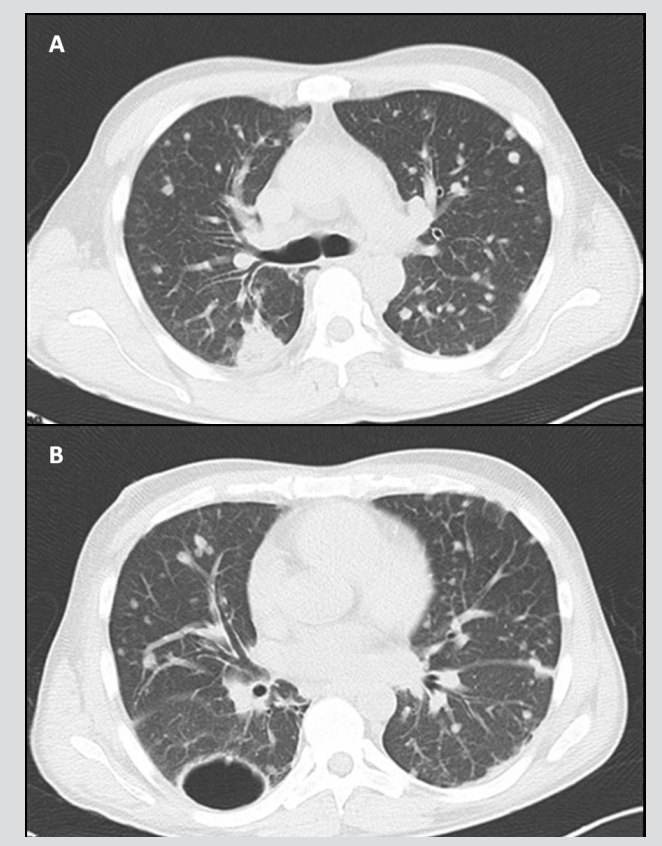

Figure 1. Thoracic CT: A and B. Multiple pulmonary nodules and nodular condensation with spiculated margins and eccentric cavitation, acknowledged as probable primary tumor

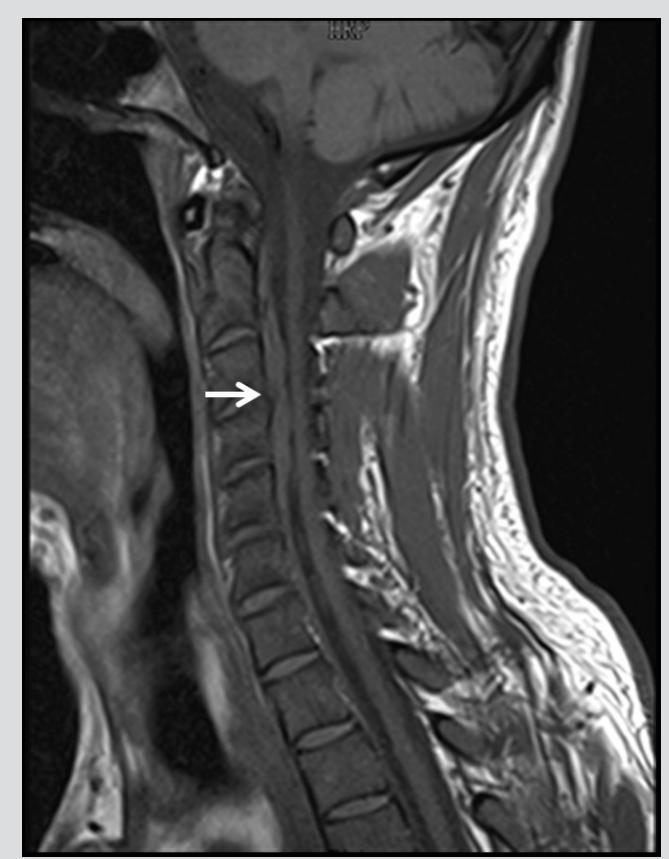

Figure 2. Cervical MRI: findings suggestive of secondary epidural infiltration (arrow) 
Imaging findings appeared unequivocal, suggesting cancer of unknown primary origin (probable lung) with pulmonary, hepatic and epidural secondary involvement. Nevertheless, the patient's previous history prompted the exclusion of pulmonary tuberculosis. Bronchofibroscopy revealed diffuse inflammatory signs along the mucosa with no evidence of cancer cells or acid-alcohol resistant bacilli (AARB). An abdominal and pelvic CT scan was performed, showing multiple focal hypodense lesions with fluid collections and a peripheral nodular enhancement pattern, typical tomodensitometric findings of liver abscesses (Fig. 3).

The patient remained on empiric antimicrobial treatment and, within a few days, his fever resolved, his cervicalgia significantly improved, and inflammatory biomarkers continuously decreased. Four weeks after admission, following discussion with Radiology, a thoracic CT scan was performed for subsequent biopsy of one of the pulmonary lesions. However, tomographic re-evaluation showed undeniable reduction in the number and volume of pulmonary nodules, with stability of the liver abscesses (Fig. 4). In light of these findings, a biopsy was not performed.

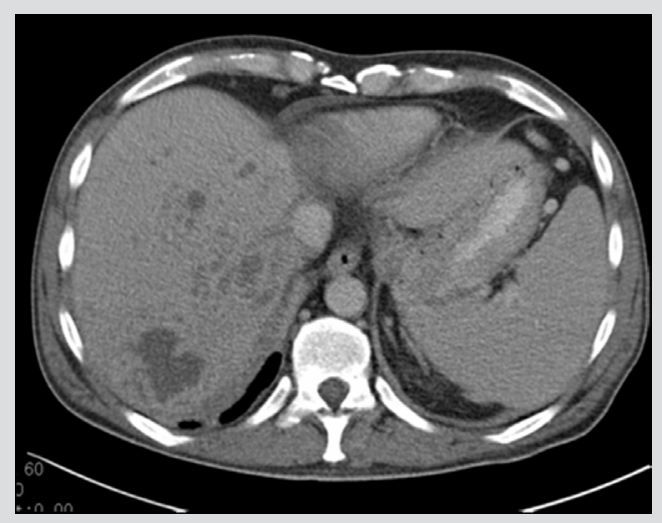

Figure 3. Abdominal CT: multiple hypodense liver lesions compatible with hepatic abscesses vs secondary necrotizing deposits

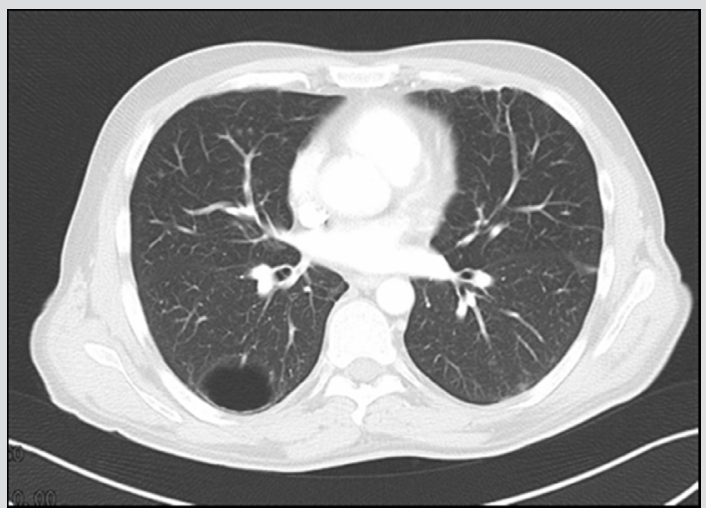

Figure 4. Thoracic CT: 4th-week follow-up with unequivocal reduction in both number and volume of nodular pulmonary lesions

This tomographic evolution thus revealed the true inflammatory/infectious nature of those pulmonary lesions, contradicting the initial hypothesis. Concurrently, the blood cultures withdrawn at admission became positive for K. pneumoniae, also isolated in bronchoalveolar lavage. Considering the clinical evolution and the microbiological isolates, a diagnosis of $K$. pneumoniae primary liver abscess with metastatic infection - best known in the literature as Klebsiella pneumoniae invasive syndrome - was made. As expected, the antibiotic susceptibility test proved the strain to be sensitive to most antibiotics (Table 2). Hence, azithromycin was stopped, while amoxicillin/clavulanic acid were continued. The patient continued to improve under antimicrobial therapy and was discharged after 6 weeks of treatment. Follow-up imaging studies showed outstanding improvement.

\begin{tabular}{|c|c|}
\hline Bronchofibroscopy & Cervical spine CT \\
\hline \multirow{2}{*}{$\begin{array}{l}\text { Cultures } \\
\qquad \begin{array}{l}\text { - Blood } \\
\text { - Bronchoalveolar lavage }\end{array}\end{array}$} & Head CT \\
\hline & Pelvic CT \\
\hline \multirow{2}{*}{$\begin{array}{l}\text { Cerebrospinal fluid analysis } \\
\text { - Leukocytes } \\
\text { - Proteins } \\
\text { - Glucose } \\
\text { - Lactate dehydrogenase }\end{array}$} & Thoracic CT \\
\hline & \multirow{6}{*}{ Table 1. Complementary diagnostic tests } \\
\hline Transthoracic echocardiogram & \\
\hline Acid-alcohol resistant bacilli (AARB) & \\
\hline Cervical MRI & \\
\hline $\begin{array}{c}\text { Viral serologies (serum) } \\
\text { - HIV } 1 \text { and } 2 \\
- \text { HBV } \\
-H C V \\
\end{array}$ & \\
\hline Abdominal CT & \\
\hline
\end{tabular}

\begin{tabular}{|l|}
\hline Susceptible \\
\hline Amoxicillin/clavulanic acid \\
\hline Cefuroxime \\
\hline Ciprofloxacin \\
\hline Meropenem \\
\hline Piperacillin/tazobactam \\
\hline Resistant \\
\hline Ampicillin \\
\hline
\end{tabular}




\section{DISCUSSION}

KPIS was diagnosed, even though neoplasia and tuberculosis were first considered. In this case, the main difficulty in establishing the diagnosis was the lack of clinical suspicion for this syndrome given its low incidence in Europe. The patient presented with a prolonged febrile illness without any relevant epidemiological context. The first imaging scans appeared unequivocal, categorically pointing to pulmonary and hepatic metastases. The vital impact of malignant neoplasms prompted the exclusion of malignancy. Nevertheless, pulmonary tuberculosis had to be considered in the differential given the patient's personal history and its high incidence in Portugal.

The unpredicted evolution of tomographic findings in conjunction with the patient's clinical improvement under antibiotic therapy and later isolation of $K$. pneumoniae in cultures were decisive in altering our clinical judgment.

The medical literature clearly points to third and fourth-generation cephalosporins as the antibiotic treatment of choice in these settings, whereas these hypermucoviscous strains have been found to be uniformly resistant to ampicillin. The treatment regimen duration varies from 2 to 4 weeks with a solitary abscess and at least 6 weeks in the event of multiple abscesses.

KPIS is nowadays a well-recognised clinical entity in Southeast Asia. For the past three decades, there has been an increasing number of reported cases in other geographic locations, in previously healthy subjects, accounting for its emergence at a global level.

In summary, the case report here illustrates just how much this entity can mimic other well-known and more common illnesses. Increased awareness of this disease could allow for earlier detection and treatment of patients, even in the absence of previous journeys to the Asian continent.

\section{REFERENCES}

1. Liu YC, Cheng DL, Lin CL. Klebsiella pneumoniae liver abscess associated with septic endophthalmitis. Arch Intern Med 1986;146:1913-16.

2. Chang FY, Chou MY, Fan RL, Shaio MF. A clinical study of Klebsiella liver abscess. Taiwan Yi Xue Hui Za Zhi 1988;87:282-87.

3. Wang JH, Liu YC, Lee SS, Yen MY, Chen YS, Wang JH, et al. Primary liver abscess due to Klebsiella pneumoniae in Taiwan. Clin Infect Dis 1998;26:1434-8.

4. Siu LK, Yeh KM, Lin JC, Fung CP, Chang FY. Klebsiella pneumoniae liver abscess: a new invasive syndrome. Lancet Infect Dis 2012;12:881-87.

5. Moore R, O'Shea D, Geoghegan T, Mallon PW, Sheehan G. Community-acquired Klebsiella pneumoniae liver abscess: an emerging infection in Ireland and Europe. Infection 2013;41:681-86. 